\title{
CONTRIBUIÇÃO AO ESTUDO ANATÔMICO DO RETALHO VASCULARIZADO CORTICOPERIOSTEAL DO CÔNDILO MEDIAL DO FÊMUR
}

\author{
CONTRIBUTION TO THE ANATOMICAL STUDY OF THE CORTICOPERIOSTEAL \\ FLAP OF THE MEDIAL FEMORAL CONDYLE
}

Rômulo Guimarães Andrade', Henrique Gubert Bufáiçal' ${ }^{1}$ Leandro Alves de Oliveira', Fabiano Inácio de Souza², Mário Yoshihide Kuwae ${ }^{3}$, João Alírio Teixeira da Silva Júnior ${ }^{4}$

\section{RESUMO}

Objetivo: Realizar o estudo anatômico, em cadáveres, do retalho corticoperiosteal do côndilo femoral medial baseado na artéria genicular medial, avaliando a dificuldade de dissecção e padrões topográficos. Métodos: Foram estudados 15 membros de oito cadáveres, com idade variando de 19 a 74 anos. Dispostos em posição supina, foi realizada incisão longitudinal na face medial do terço distal da coxa, exposição dos vasos geniculares descendentes entre os músculos vasto medial e sartório. Foram analisados a distância entre a origem da artéria genicular descendente e a interlinha medial do joelho, o diâmetro do vaso, o comprimento do pedículo, a presença do ramo fasciocutâneo e sua localização. Resultados: A distância entre a origem da artéria genicular descendente e a interlinha medial do joelho variou de $11,2 \mathrm{~cm}$ a $14,5 \mathrm{~cm}$, com média de $12,63 \mathrm{~cm}$. O diâmetro médio da artéria foi de $2,5 \mathrm{~mm}$ (de $2,25 \mathrm{~mm}$ a $2,75 \mathrm{~mm}$ ). A distância entre a origem da artéria genicular descendente e o ramo fasciocutâneo variou entre 1,0 e $1,5 \mathrm{~cm}$. O comprimento médio do pedículo vascular, foi de $7,01 \mathrm{~cm}$, variando de 5,6 a $8,6 \mathrm{~cm}$. Conclusão: O retalho corticoperiosteal do côndilo femoral medial do joelho é de fácil dissecção, possui pedículo vascular constante, com comprimento médio de $7,0 \mathrm{~cm}$ e diâmetro de $2,5 \mathrm{~mm}$, o que possibilita sua indicação em transplantes microcirúrgicos.

Descritores - Cadáver; Anatomia; Microcirurgia

\section{ABSTRACT}

Objective: to perform the anatomical study, in cadavers, of the corticoperiosteal flap of the medial femoral condyle, based on the medial genicular artery, evaluating challenges in dissection and the topographic patterns. Materials and methods: fifteen limbs from eight cadavers were studied, ages ranging from 19 to 74 years old. They were placed at supine position, and a longitudinal incision on the medial face of the lower part of the thigh was performed, exposing medial vastus and sartorius muscles, with descendent genicular vessels being also exposed. The distance between the descendent genicular artery and the medial articular line of the knee, the diameter of the vessel, the length of the pedicle, and the presence of the fasciocutaneous branch and its location were analyzed. Results: the distance between the origin of the descendent genicular artery and the medial articular line of the knee ranged from $11.2 \mathrm{~cm}$ to $14.5 \mathrm{~cm}$, with an average of $12.63 \mathrm{~cm}$. The mean artery diameter was $2.5 \mathrm{~mm}$ (from $2.25 \mathrm{~mm}$ to $2.75 \mathrm{~mm}$ ). The distance between the descendent genicular artery and the fasciocutaneous branch ranged from 1.0 to $1.5 \mathrm{~cm}$. The mean length of the vascular pedicle was $7.01 \mathrm{~cm}$, ranging from $5.6 \mathrm{~cm}$ to $8.6 \mathrm{~cm}$. Conclusion: the corticoperiosteal flap of the medial femoral condyle of the knee is easy to dissect, presents a constant vascular pedicle, with average length of $7.0 \mathrm{~cm}$ and diameter of $2.5 \mathrm{~mm}$, enabling it to be indicated for microsurgical transplants.

Keywords - Cadaver; Anatomy; Microsurgery

\footnotetext{
1 - Médico Residente do $3^{\circ}$ ano de Ortopedia e Traumatologia do HC-FM-UFG, Goiás, Brasil.

2 - Mestre em Ortopedia pelo Departamento de Ortopedia da Faculdade de Medicina da USP, Goiás, Brasil.

3 - Chefe do Grupo de Mão e Microcirurgia do Departamento de Ortopedia do HC-FM-UFG, Goiás, Brasil.

4 - Chefe do Departamento de Ortopedia, Traumatologia e Cirurgia Plástica do HC-FM-UFG, Goiás, Brasil.
}

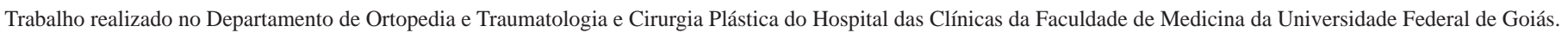

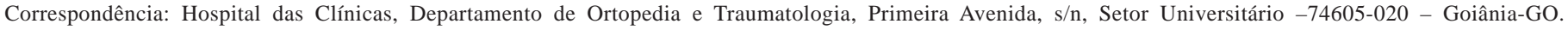
E-mail: fabianoinacio@hotmail.com 


\section{INTRODUÇÃO}

As lesões traumáticas dos membros superiores e inferiores podem ocasionar inúmeras sequelas, dentre elas as pseudoartroses, que são passíveis de grandes dificuldades no seu tratamento.

Existem vários métodos para o tratamento das pseudoartroses, desde a utilização de materiais de síntese como placas de compressão, inúmeras tipos de fixadores externos, encavilhamento intramedular, geralmente associados a enxertos ósseos avasculares, obtendo-se sucesso no tratamento na maioria dos casos.

Nos pacientes que não evoluem satisfatoriamente, podem-se indicar os enxertos ósseos vascularizados, como proposto por Östrup e Fredrickson ${ }^{(1)}$, obtendo-se bons resultados, sendo o enxerto de fíbula vascularizada o mais utilizado, embora seja de difícil dissecção e com potencial de causar instabilidade no segmento doador ${ }^{(1,2)}$

O objetivo deste trabalho é o estudo anatômico, em cadáveres, do retalho corticoperiosteal do côndilo femoral medial baseado na artéria genicular medial, avaliando a dificuldade de dissecção e padrões topográficos.

\section{MÉTODOS}

Foram utilizados para a dissecção 15 membros (oito cadáveres), provenientes do Instituto de Medicina Legal do Estado de Goiás, no período entre os meses de abril e julho de 2007.

A idade dos cadáveres variou entre 19 e 74 anos, sendo cinco do sexo masculino e três do feminino e altura entre $1,64 \mathrm{~m}$ e $1,83 \mathrm{~m}$.

Os cadáveres foram dispostos em posição supina, com um dos membros inferiores em rotação externa (Figura 1). Foi realizada uma incisão longitudinal na face medial do terço inferior da coxa (Figura 2). A fáscia foi incisada no mesmo sentido, expondo os músculos vasto medial (rebatido anteriormente), o sartório (rebatido posteriormente), sendo visibilizados os vasos geniculares descendentes (Figuras 3 e 4). Esses vasos emergem da artéria femoral, distal ao hiato adutor, e ramificam-se sobre o periósteo do côndilo femoral medial (Figura 5).

Foram mensurados: a distância entre a origem da artéria genicular descendente e a interlinha medial do joelho, o diâmetro do vaso, o comprimento do pedículo, a presença do ramo fasciocutâneo e sua localização. Para a obtenção dessas medidas, foi utilizado um escalímetro (Trident ${ }^{\circledR} \mathrm{n}^{\circ} 2$, modelo 7830/1).

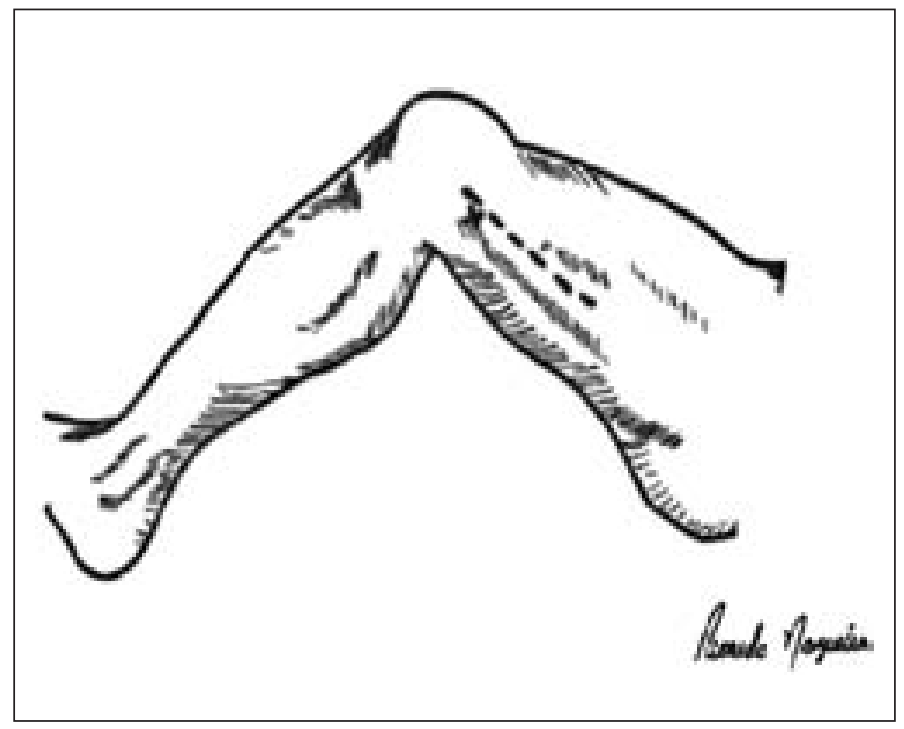

Figura 1 - Posicionamento do membro e local da incisão

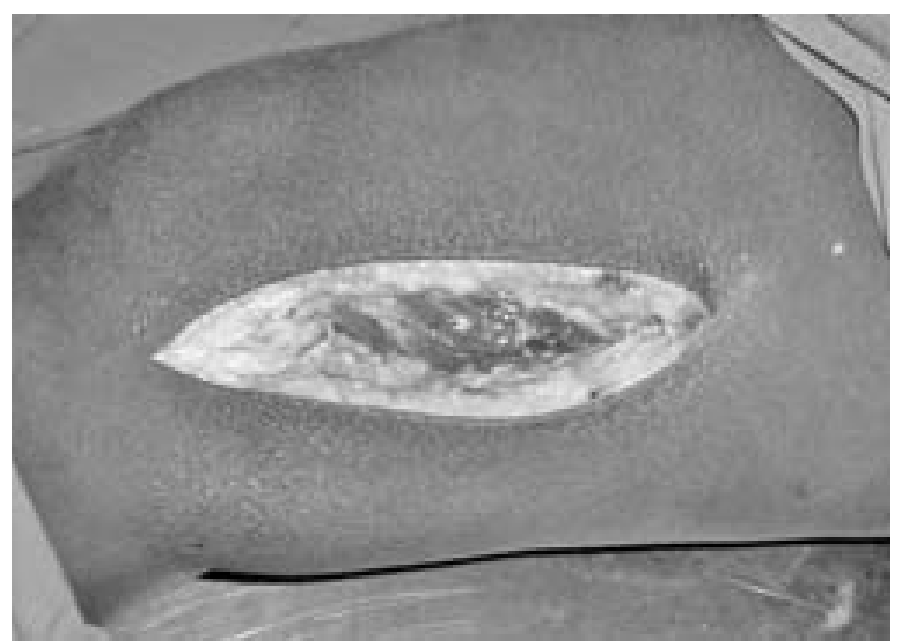

Figura 2 - Incisão na pele e subcutâneo

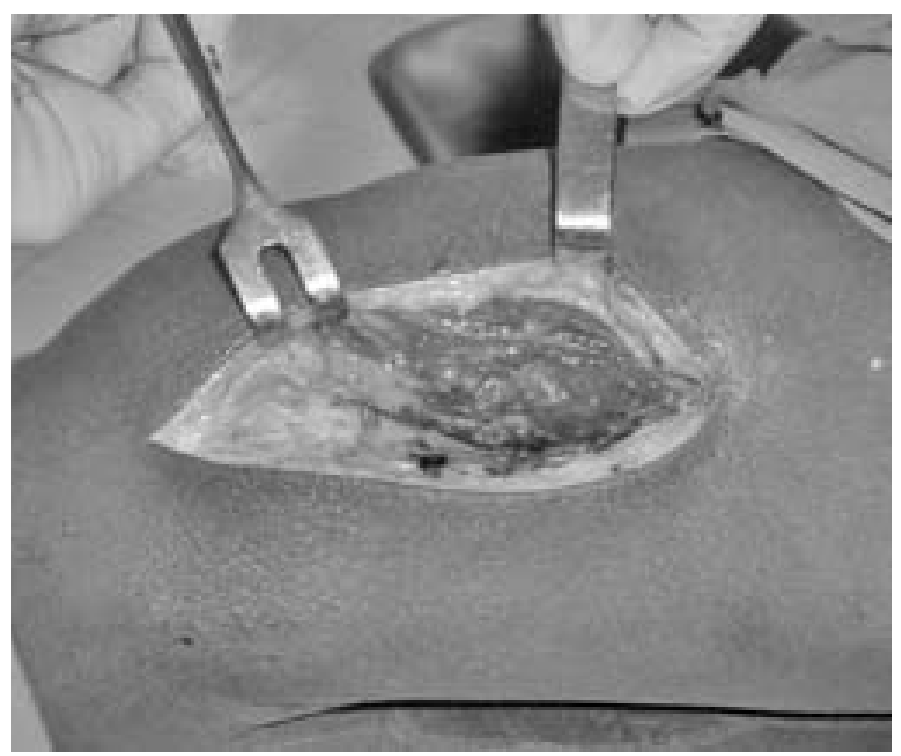

Figura 3 - Afastamento anterior do vasto medial 


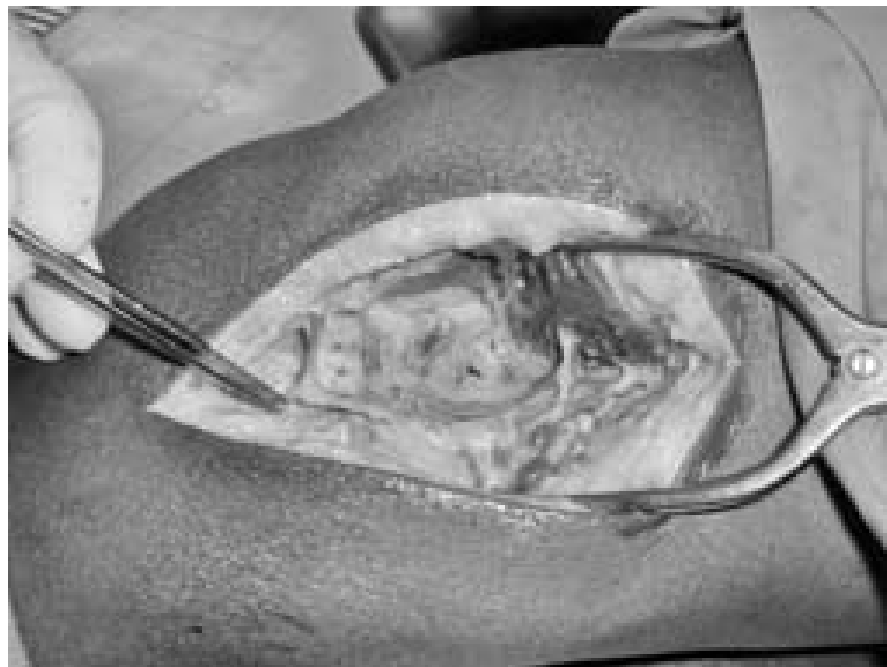

Figura 4 - Exposição do pedículo e seus ramos

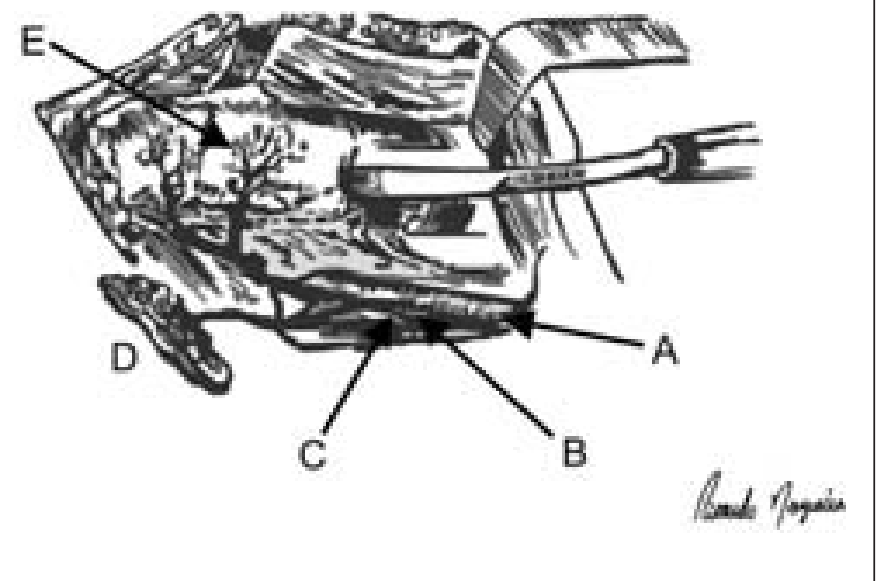

Figura 5 - Esquema das estruturas anatômicas. A: artéria femoral. B: origem da artéria genicular. C: tendão do adutor magno. D: ramo fasciocutâneo

\section{RESULTADOS}

A média da altura dos cadáveres foi de $1,67 \mathrm{~m}$ para 0 sexo feminino (variando de $1,64 \mathrm{~m}$ a $1,71 \mathrm{~m}$ ) e de $1,75 \mathrm{~m}$ para o masculino $(1,68 \mathrm{~m}$ a $1,83 \mathrm{~m})$.

A distância entre a origem da artéria genicular descendente e a interlinha medial do joelho variou de $11,2 \mathrm{~cm}$ a $14,5 \mathrm{~cm}$, com média de $12,63 \mathrm{~cm}$. O diâmetro médio da artéria foi de 2,5mm (de 2,25mm a 2,75mm). A distância entre a origem da artéria genicular descendente e o ramo fasciocutâneo variou entre 1,0 e 1,5cm. Esse ramo esteve ausente em dois casos. O comprimento médio do pedículo vascular, compreendido entre a artéria femoral e o periósteo do côndilo medial, foi de $7,01 \mathrm{~cm}$, variando de 5,6 a $8,4 \mathrm{~cm}$ (Tabela 1 ).
Tabela 1 - Mensuração dos dados extraídos

\begin{tabular}{|c|c|c|c|c|c|}
\hline Membro & Altura (m) & $\begin{array}{c}\text { Distância entre } \\
\text { artéria genicular } \\
\text { e interlinha } \\
\text { medial (cm) }\end{array}$ & $\begin{array}{l}\text { Diâmetro do } \\
\text { vaso (mm) }\end{array}$ & $\begin{array}{l}\text { Origem do } \\
\text { ramo cutâneo } \\
\text { (cm) }\end{array}$ & $\begin{array}{c}\text { Comprimento } \\
\text { do pedículo } \\
\text { (cm) }\end{array}$ \\
\hline 1 & 1,64 & 11,2 & 2,5 & 1,2 & 5,6 \\
\hline 2 & 1,64 & 11,3 & 2,5 & 1,1 & 5,8 \\
\hline 3 & 1,67 & 11,7 & 2,25 & 1,1 & 6,1 \\
\hline 4 & 1,67 & 11,7 & 2,5 & 1,3 & 6,2 \\
\hline 5 & 1,71 & 12,3 & 2,5 & 1,2 & 6,3 \\
\hline 6 & 1,71 & 12,5 & 2,5 & 1,3 & 6,4 \\
\hline 7 & 1,68 & 12,1 & 2,5 & 1,0 & 6,1 \\
\hline 8 & 1,68 & 11,9 & 2,25 & Ausente & 6,0 \\
\hline 9 & 1,72 & 12,5 & 2,5 & 1,4 & 7,5 \\
\hline 10 & 1,72 & 12,9 & 2,75 & 1,1 & 7,6 \\
\hline 11 & 1,75 & 13,6 & 2,75 & Ausente & 8,3 \\
\hline 12 & 1,75 & 13,4 & 2,25 & 1,3 & 8,4 \\
\hline 13 & 1,79 & 13,8 & 2,75 & 1,4 & 7,9 \\
\hline 14 & 1,79 & 14,1 & 2,5 & 1,3 & 8,4 \\
\hline 15 & 1,83 & 14,5 & 2,5 & 1,5 & 8,6 \\
\hline
\end{tabular}

Fonte: DOT-HC-FMUFG

\section{DISCUSSÃO}

Baseados nos estudos de Östrup e Fredrickson ${ }^{(1)}$, e Finley et al $^{(2)}$, que demonstravam boa capacidade osteogênica dos retalhos microcirúrgicos ósseos e periosteais, Sakai et $\mathrm{al}^{(4)}$ propuseram um novo retalho corticoperiosteal, dissecado nas regiões condilares e supracondilares mediais do fêmur.

Em 1989, Masquelet et al $^{(5)}$ publicam as bases anatômicas dos retalhos periosteais, descrevendo a região do côndilo femoral medial como possível sítio doador. O retalho corticoperiosteal do côndilo medial do joelho é baseado no feixe vascular genicular medial, ramo da artéria femoral. Neste estudo, o diâmetro médio da artéria foi de $2,5 \mathrm{~mm}$, encontrando-se dentro dos limites de 1,5 e 3,8 , relatados por outros autores ${ }^{(4,6,7)}$.

O comprimento do pedículo também assegura a viabilidade do procedimento, pois a média do seu comprimento $(7,0 \mathrm{~cm})$ proporciona alternativas de mobilidade e disposição espacial na área receptora.

A origem constante da artéria genicular medial, com distância média de $12,63 \mathrm{~cm}$ da interlinha medial do joelho, confere segurança na execução do planejamento pré-operatório. 
Esses dados encontram-se de acordo com a literatura. Porém, outros autores não mencionam a disposição anatômica do ramo fasciocutâneo emitido pela artéria genicular medial. Esse ramo emerge, em $86,6 \%$ dos casos, entre 1,0 e $1,5 \mathrm{~cm}$ da origem da artéria genicular. Em dois casos $(13,4 \%)$ o ramo fasciocutâneo emergiu diretamente da artéria femoral.

A importância do conhecimento desse ramo decorre da possibilidade de inclusão de um segmento fasciocutâneo no retalho corticoperiosteal, onde poderia ser utilizado como monitor de pele ou para estabilizar partes moles adjacentes.

A aplicação clínica do retalho corticoperiosteal do côndilo femoral medial é descrita por alguns cirurgiões no tratamento de diversos tipos de pseudoartroses.

Enquanto Doi et al $^{(6)}$ demonstraram $80 \%$ de bons resultados em pseudoartroses de escafoide, Kobayashi et $a l^{(8)}$ relataram resultados favoráveis em lesões periorbitárias.

Já Fuchs et $a l^{(9)}$ obtiveram bons resultados no tratamento de pacientes portadores de pseudoartrose de clavícula e ressaltam que o emprego desse retalho vascularizado é tecnicamente mais fácil, rápido e com menor morbidade na área doadora, quando comparado com o fibular.

Há relatos de bons resultados também no tratamento de pseudoartroses de úmero distal ${ }^{(10)}$ e tíbia distal ${ }^{(11)}$.

Choudry et $a^{(12)}$ enfatizam que o retalho corticoperiosteal do côndilo femoral medial é a melhor indicação no tratamento de pseudoartroses recorrentes menores que $5,0 \mathrm{~cm}$.

O conhecimento anatômico é a base para se obter maior segurança nos procedimentos cirúrgicos, principalmente em técnicas promissoras e pouco difundidas.

\section{CONCLUSÃO}

O retalho corticoperiosteal do côndilo femoral medial do joelho é de fácil dissecção, possui pedículo vascular constante, com comprimento médio de $7,0 \mathrm{~cm}$ e diâmetro de 2,5mm, o que possibilita sua indicação em transplantes microcirúrgicos.

\section{REFERÊNCIAS}

1. Östrup LT, Fredrickson JM. Distant transfer of a free living bone graft by microvascular anastomoses: an experimental study. Plast Reconstr Surg. 1974;54(3):274-85.

2. Finley JM, Acland RD, Wood MB. Revascularized periosteal grafts: A new method to produce functional new bone without bone grafting. Plast Reconstr Surg. 1978;61(1):1-6.

3. Sakai K, Doi K, Tamaru K, Yamamoto M, Kawai S. Free Vascularized bone and periosteal graft for pseudoarthrosis in the upper limb. J Jpn Soc Surg Hand. 1988;5:698-704.

4. Sakai K, Doi K, Kawai S. Free vascularized thin corticoperiosteal graft. Plast Reconstr Surg. 1991;87(2):290-8.

5. Masquelet AC, Penteado CV, Romana MC, Chevrel JP. Periosteal flaps: anatomical bases of sites of elevation. Surg Radiol Anat. 1990;12(1):3-7.

6. Doi K, Oda T, Soo-Heong T, Nanda V. Free vascularized bone graft for nonunion of the scaphoid. J Hand Surg Am. 2000;25(3):507-19.

7. Masquelet AC, Hertel R. The reverse flow medial knee osteoperiosteal flap for skeletal reconstruction of the leg. Description and anatomical basis. Surg Radiol Anat. 1989;11(4):257-62

8. Kobayashi S, Kakibuchi M, Masuda T, Ohmori K. Use of vascularized corticoperiosteal flap from the femur for reconstruction of the orbit. Ann Plast Surg. 1994;33(4):351-7.

9. Fuchs B, Steinman SP, Bishop AT. Free vascularized corticoperiosteal bone graft for the treatment of persistent nonunion of the clavicle. J Shoulder Elbow Surg. 2005;14(3):264-8.

10. Kakinoki R, Ikeguchi R, Nakayama K, Yamakawa T, Morimoto M, Nakamura T. Treatment of avascular necrosis of the capitelum of the humerus using a free vascularized osteoperiosteal graft from the medial condyle of the femur: a case report. J Shoulder Elbow Surg. 2008;17(1):e1-4.

11. Cavadas PC, Landin L. Treatment of recalcitrant distal tibial nonunion using the descending genicular corticoperiosteal free flap. J Trauma. 2008;64(1):144-50.

12. Choudry UH, Bakri K, Moran SL, Karacor Z. Shin AY. The vascularized medial femoral condyle periosteal bone flap for the treatment of recalcitrant bony nonunion. Ann Plast Surg. 2008;60(2):174-80. 